\title{
Mapping of index of refraction profile for polymer gradient index optics using confocal Raman spectroscopy
}

\author{
Nicholas S. Kochan,* Greg R. Schmidt, Andrew J. Berger, and \\ Duncan T. Moore \\ University of Rochester, The Institute of Optics, Rochester, New York, United States
}

\begin{abstract}
Scanning confocal Raman microscopy is proposed to measure a gradient index (GRIN) profile at an optical surface. The Raman microscope is calibrated to index of refraction for a binary copolymer GRIN material, and then the index of refraction is mapped on the plano surface of a GRIN polymer lens. The measurement deduces axial shift of $680 \mu \mathrm{m}$ and identifies lateral tilt or decenter with respect to the nominal position of the GRIN profile. Results suggest that the mapping method is a nondestructive way to measure the GRIN profile of a GRIN lens and its positioning within the lens geometry, to within the sampling precision of the Raman microscope. () The Authors. Published by SPIE under a Creative Commons Attribution 4.0 Unported License. Distribution or reproduction of this work in whole or in part requires full attribution of the original publication, including its DOI. [DOI: 10.1117/1.OE.59.11.112605]
\end{abstract}

Keywords: gradient index; optics; metrology; nondestructive measurement; Raman spectroscopy; confocal microscopy.

Paper 20200301SS received Mar. 13, 2020; accepted for publication Jun. 16, 2020; published online Jul. 2, 2020.

\section{Introduction}

Gradient index (GRIN) optics offer many advantages in optical design but often pose unique optical metrology challenges. Direct measurements of imaging performance at the design wavelengths, such as with interferometry, ${ }^{1}$ can be used for measuring effects of manufacturing errors in a GRIN lens system. However, these effects on imaging performance cannot diagnose all errors of GRIN fabrication. To this end, Raman spectroscopy can provide material distribution information, and therefore index of refraction information, to measure the optic nondestructively. Raman spectroscopy is superior to interferometry for mapping the GRIN profile in the following ways: it can nondestructively measure large differences in index of refraction and can map the absolute index of refraction rather than difference in index of refraction across the optic. The accuracy of the index of refraction map is limited by the ability to map Raman spectrum to index of refraction, which can be ensured with proper spectrum calibration of the material used for the GRIN optic. This work outlines the mapping procedure.

For a binary material GRIN optic, Raman spectroscopy serves as a suitable candidate measurement method. The objective of the present work is to map index of refraction in a polystyrene (PS)/poly(methyl methacrylate) (PMMA) copolymer GRIN lens and report accuracy of mapping the profile within the lens (Fig. 1). This is important because the lens is cut out of a large blank of GRIN material. During the diamond turning of the optical surfaces, the GRIN coordinate system may end up shifted in the lens geometry. Because of the large index of refraction variation through the lens presently under test, Fang ${ }^{1}$ took a plane-parallel radial slice of a GRIN lens of similar design [as in Fig. 1(d)]. This slice was used to measure the GRIN profile in a Mach-Zehnder interferometer. Slicing the sample reduced optical path length to produce a two-dimensional (2-D) index of refraction map with interferometry, but destroyed the lens. Using Raman microscopy, alignment of the GRIN profile within this GRIN lens is measured nondestructively.

*Address all correspondence to Nicholas S. Kochan, E-mail: nkochan@u.rochester.edu 
(a)

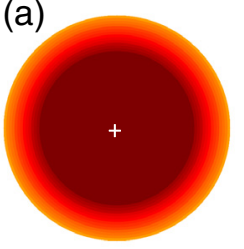

(d)

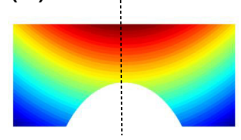

(b)

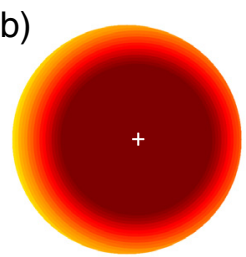

(e)

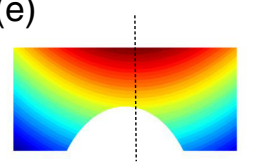

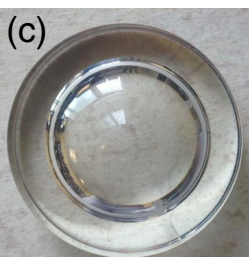

(f)

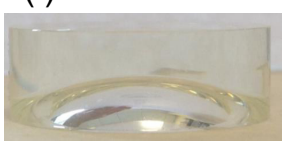

$\leftarrow$ Index of refraction

High

Fig. 1 Schematic of the GRIN lens: (a)-(c) top surface and (d)-(f) diametric cut section views are presented of GRIN profile schematic (a), (b), (d), (e) and of the fabricated lens (c), (f). The fabricated GRIN profile (b), (e) may be decentered from the designed GRIN profile (a), (d) by some amount. Cross and dotted line indicates optical axis relative to the GRIN profile.

Section 1 outlines GRIN optics demanding better metrology and the corresponding capabilities in Raman spectroscopy. Section 2 provides the GRIN design under test and the methods to relate index of refraction to Raman spectra. Section 3 provides the scan results and indication of a shifted GRIN profile within the lens, and Sec. 4 provides interpretation of the results and suggestions for the future directions of this work.

\subsection{Geometries of Gradient Index Optics}

Practical application of GRIN optics relies on the ability to fabricate the GRIN to design tolerances and the ability to measure the GRIN profile. The first commercially available solid-state GRIN optics were glass radial GRIN rod lenses, under the trade name SELFOC. ${ }^{2}$ Periodic focusing and collimation within a single radial GRIN lens give it a variety of cited applications in fiber communications, ${ }^{3,4}$ biomedical sensing,${ }^{5,6}$ and imaging. ${ }^{7,8}$

For most common GRIN rods with radial symmetry, the index of refraction changes only as a function of radial distance from a linear axis. One common way of making a glass radial GRIN lens is by ion diffusion ${ }^{7,8}$ through the boundaries of a glass rod. The glass rod preform exhibits rotational invariance; therefore, the resultant GRIN profile is also rotationally invariant because the ions diffuse radially across the rod surface.

Another type of gradient employed is the axial GRIN optic, where the index of refraction changes only as a function of one spatial axis. This index of refraction distribution is also rotationally invariant about an axis, usually the optical axis. Such a lens can be used for beam shaping ${ }^{9}$ and aberration correction. ${ }^{10}$ For both of the rotationally invariant forms, assessing the tilt of the GRIN is necessary to correctly tolerance the GRIN lens performance.

The axial and radial GRIN geometries are common, and they both respect rotational symmetry to easily interface with rotationally symmetric homogeneous lens systems. Furthermore, their symmetries often are limited to their manufacturing technique. Recent developments in freeform optics have influenced the practical design and testing of nonrotationally symmetric optical surfaces ${ }^{11}$ comprising homogeneous material lens systems. Simultaneously, developments in three-dimensional (3-D) printing of optics have enabled deterministic freefrom GRIN fabrication techniques. ${ }^{12}$ The main limitation to freeform GRIN fabrication is that designed freeform and GRIN optics often possess only a weak departure from a rotationally invariant design. For homogeneous freeforms, this departure is designed large enough to correct aberration but small enough to not heavily impact first-order optics, making the freeform surfaces suitable for deterministic nulling interferometry. ${ }^{11}$ 
In contrast, lack of readily available tools for nondestructive measurement of nonrotationally symmetric strongly refracting GRIN optics motivates this work. Ellipsometry has acheived promising measurements in bulk radial glass GRIN ${ }^{13}$ and in axial gradient thin films. ${ }^{14}$ However, the diamond turning of the surfaces on the currently tested polymer GRIN lens did not achieve the surface quality necessary for desired precision index of refraction measurement using ellipsometry. In an interferometer, measurement of strong refracting profiles may be limited by high-fringe density. Other methods to measure GRIN include laser beam deflectometry ${ }^{15,16}$ and fringe projection deflectometry ${ }^{17}$ techniques. These techniques assume geometries such as axial GRIN ${ }^{15,17}$ or 2-D GRIN, ${ }^{16}$ making them unsuitable for measuring some asymmetric profile departures in 3-D space. Methods such as spectroscopy leverage nonlinear optical response to effectively measure freeform variation of the GRIN profile.

\subsection{Raman Spectroscopy Impact on Polymer GRIN Optics}

Measurement of the index of refraction at a flat surface of a homogeneous lens can be obtained using a refractometer such as the Abbe refractometer. When the index of refraction varies along a surface, conventional refractometry is no longer suitable.

Ellipsometry is often used to measure index of refraction at an interface, however, confocal Raman spectroscopy is better suited to the current application. Interference effects localized to the diamond-turned optical surface may confound the ellipsometric measurement of the GRIN profile, but not the Raman measurement. For this reason, Raman measurement is pursued for the current polymer lens.

The Raman signal must be properly extracted for effective measurements. Raman spectra contain material-characteristic information in sharp peaks, which must be distinguished from broader spectrum fluorescence features. ${ }^{18}$ Fluorescence is reduced by using a relatively long excitation wavelength for the Raman spectrum, and polymers are good candidates for Raman spectroscopy because of their low-moisture content and narrowness of their Raman spectral signature. Low moisture reduces fluorescence in the signal, and narrow Raman spectral peaks improve the ability to distinguish materials.

Raman microscopy has been applied to measure the diffusion of a PMMA/PS binary mixture film by Hu et al. ${ }^{19}$ The authors analyzed the profile of a few characteristic Raman spectral lines along the confocal scan. The authors mentioned that the difference in the index of refraction of the sample would amount to distortion in the focal volume of the microscope and emission of Raman scattered light. Commentary on the work elaborated the need to correct for aberrations due to the refraction of the spot being collected by the dry objective of a Raman microscope. ${ }^{20}$ These effects were analyzed by Tomba et al. ${ }^{21}$ and Everall ${ }^{22}$ and certainly would need to be considered for some geometries of bulk GRIN optics. For a binary copolymer GRIN profile measurement, either a set of reference measurements should be taken to account for the results of different diffractions and aberration-induced Raman scattering volumes, or the Raman microscope system should be empirically modeled, accounting for focal volume refraction and diffraction. Calibration by reference measurements of known binary copolymer constituent concentrations generates a type of lookup table, and empirical modeling requires meticulous instrument calibration. For proof of concept on an existing lab instrument, the lookup table method is most approachable, especially when the index of refraction of the GRIN sample varies minimally within the focal spot.

Raman spectroscopy has been applied to GRIN for a variety of materials. The degree of two-photon photopolymerization for GRIN materials has been measured by Raman microscopy. ${ }^{23}$ Raman spectroscopy was briefly mentioned as a validation method to measure the mixture of styrene acrylonitrile, PMMA, and poly(vinylidene fluoride-cotrifluoroethylene) for application in GRIN. ${ }^{24}$ Raman spectroscopy has also been applied to measure infrared ceramic GRIN ${ }^{25}$ and infrared glass GRIN ${ }^{26,27}$ optics. In one of these works, Raman spectroscopy mapped the GRIN profile of the infrared glass ceramic by measuring its material morphology. ${ }^{25}$ In contrast, this work describes polymer GRIN created predominantly by binary material mixture. This work proposes a method to create a full spatial index of refraction map of a binary copolymer GRIN lens surface and determine accuracy to which GRIN is aligned withing the lens geometry. 


\section{Measurement Model and Methods}

A PMMA/PS copolymer GRIN lens fabricated at the University of Rochester has an index of refraction varying as a function of both radial and axial position (Fig. 1). Previously, the GRIN lens was measured destructively to produce a map of the relative index of refraction variation through the sample. Scanning Raman microscopy in the current study, however, can map the absolute index of refraction at a known surface.

The model for measuring the GRIN profile is to use the Raman spectrum to get a material distribution within the focal volume of the microscope and then to use the material distribution to get the index of refraction at a desired wavelength. Because the Raman microscope assesses material in a volume, variation in the focusing of the beam and free volume of the polymer may bias the measurement. These contributions are minimal relative to the measured change in index of refraction. The index of refraction change over the $1.7-\mu \mathrm{m}$ focal spot diameter, as in the setup described below, is $4 \times 10^{-5}$ index of refraction. Calibration measurements on homogeneous copolymer samples verify that the Raman spectrum and index of refraction can be linearly correlated. Details of the calculation follow below. Calibration measurements provide the precision of the Raman scan at each index of refraction value.

The method employed here uses a least squares fit to determine the best linear combination of two homogeneous polymer spectra for PS and PMMA for a measured PS/PMMA copolymer spectrum. Given known basis spectra of PS and PMMA, this solves for a $1 \times i$ coefficient vector $\mathbf{C}$, where $i$ is the number of coefficients for basis spectra under consideration. Two coefficients are for the PS and PMMA basis spectra, and these coefficients are assumed linear with volume fraction of the constituent materials. The rest of the coefficients are for polynomial orders up to fifth order in Raman frequency. These polynomial orders are used to reduce the effect of any spectrally broad fluorescence signal on the measurement fit to PS and PMMA basis spectra. Each coefficient vector element weights the contribution of each basis spectrum to the measured $1 \times j$ spectrum vector $\mathbf{M}$, where $j$ is the number of discrete frequency measurements sampling the Raman frequency spectrum. The coefficients and measurement are related by Eq. (1), where $i \times j$ is the matrix $B$ of basis spectra and $1 \times j$ noise vector $\mathbf{N}$. Equation (1) is the model for the ordinary least squares fit:

$$
\mathbf{M}=B \boldsymbol{C}+\boldsymbol{N}
$$

from which optimal $\mathbf{C}$ is solved by psuedoinversion on the rectangular matrix $B$ to minimize the noise $\mathbf{N}$ applied to measurement $\mathbf{M}$. The psuedoinverse matrix $B^{+}$of basis spectra $B$ (with matrix transpose denoted by ${ }^{\mathrm{T}}$ ) is

$$
B^{+}=\left(B^{\mathrm{T}} B\right)^{-1} B^{\mathrm{T}} \text {. }
$$

The psuedoinverse then is used to recover optimal coefficients $\mathbf{C}$ by

$$
\mathbf{C}=B^{+} \mathbf{M}
$$

The resulting coefficients in $\mathbf{C}$ for PS and PMMA, $C_{\mathrm{PS}}$ and $C_{\mathrm{PMMA}}$, are then the weights to compute the index of refraction $n_{\text {GRIN }}$ by a weighted average of PS and PMMA index of refraction, $n_{\mathrm{PS}}$ and $n_{\mathrm{PMMA}}$ :

$$
n_{\mathrm{GRIN}}=\frac{\left(C_{\mathrm{PMMA}} n_{\mathrm{PMMA}}+C_{\mathrm{PS}} n_{\mathrm{PS}}\right)}{\left(C_{\mathrm{PMMA}}+C_{\mathrm{PS}}\right)} .
$$

To confirm that this measurement model is reasonable for spectroscopy on the GRIN copolymer lens, homogeneous copolymer PS/PMMA samples of known index of refraction and composition are used for reference. The homogeneous samples are measured on both a Pulfrich refractometer and the Raman microscope. Comparing measurements ensures that index of refraction is correlated with the different copolymer constituent concentrations. Pure PMMA and PS homopolymer spectra are measured as well: they are the endpoints of the mixing model. These pure spectra serve as basis spectra for the analysis. 
A Pulfrich refractometer built in-house was used to obtain high-accuracy reference index of refraction measurements, as in the thesis work of Fang. ${ }^{1}$ Pulfrich index of refraction measurements are taken at wavelength $\lambda=532 \mathrm{~nm}$. Therefore, all index of refraction data is reported at $\lambda=532 \mathrm{~nm}$. Scanning confocal Raman microscopy is carried out with a custom-built microscope, the construction and details of which are outlined in the thesis work of Beier. ${ }^{28} \mathrm{~A}$ basic schematic of the microscope is provided (Fig. 2). The probe laser has wavelength $\lambda=830 \mathrm{~nm}$, and the microscope objective (MO) is a $10 \times, 0.3 \mathrm{NA}$ dry objective. In air, this objective gives a lateral spot diameter of $1.7 \mu \mathrm{m}$.

The Raman microscope is scanned over the plano surface of the GRIN lens to measure the GRIN profile. To deduce any shift in the measured GRIN profile relative to the designed profile, the data must be fit to the nominal design form with some allowance for GRIN decenter and tilt. The nominal GRIN design is an eighth-order radially symmetric polynomial with radial coefficients $c_{10} \ldots c_{40}$ at the $z=0$ plane (Fig. 3). Therefore, the GRIN profile at the $z=0$ plane of the lens, accounting for possible small lateral shifts $\delta x, \delta y$ and tilts $t_{x}, t_{y}$ of the GRIN profile, takes the general form:

$$
\begin{aligned}
n(x, y)= & n_{0}+t_{x} x+t_{y} y+c_{10}\left[(x-\delta x)^{2}+(y-\delta y)^{2}\right] \\
& +c_{20}\left[(x-\delta x)^{2}+(y-\delta y)^{2}\right]^{2}+c_{30}\left[(x-\delta x)^{2}+(y-\delta y)^{2}\right]^{3} \\
& +c_{40}\left[(x-\delta x)^{2}+(y-\delta y)^{2}\right]^{4} .
\end{aligned}
$$

To account for any axial, or $z$, shift in the GRIN profile within the lens using the real data, the linear term describing the axial variation of the GRIN is used.

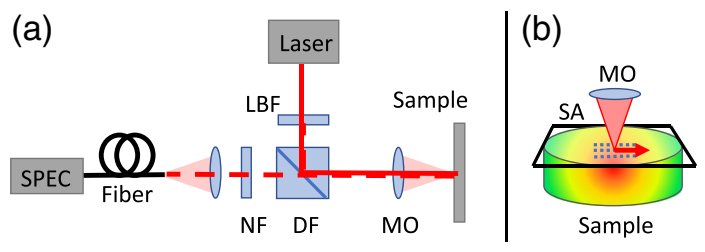

Fig. 2 Schematic of the (a) scanning confocal Raman miscroscope system and (b) scan configuration. The system (a) has probe source indicated in solid red and Raman scattered light indicated in dotted red lines. Probe laser light passes through a laser bandpass filter (LBF) and a dichroic filter (DF) to then be focused onto the sample by the microscope objective (MO). Scattered Raman light is collected by the objective, passing through the DF and through a notch filter (NF) to then be focused into optical fiber for the spectrometer. Residual reflection off the DF is blocked by the LBF. The sample scan (b) is arranged such that the sample moves under the $\mathrm{MO}$ in a raster pattern to cover the entire $25 \mathrm{~mm} \times 25 \mathrm{~mm}$ scan area enclosing the planar sample surface. Measurements are separated by $400 \mu \mathrm{m}$ in a square grid.

(a)

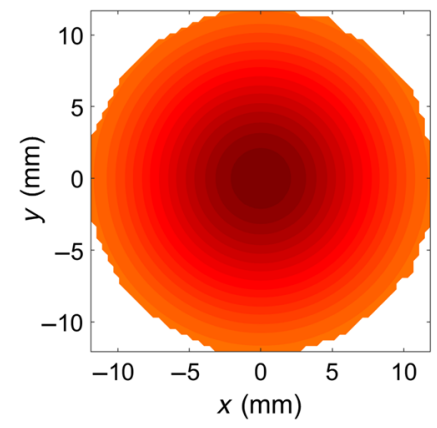

(b)

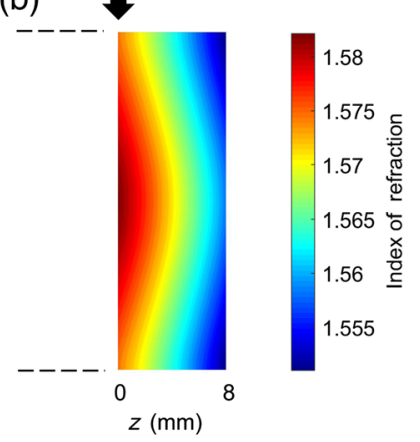

Fig. 3 Design index of refraction profile for the GRIN lens. Two plane cuts through the rotationally symmetric index of refraction design profile are shown. (a) The $X Y$ plane profile is a view of the the GRIN at the $z=0$ plane located by the black arrow in (b) the $Y Z$ plane profile. Both plots share the same index of refraction scale. 
Using the MATLAB surface fitting tool, the raw index data are fit to the above shifted form of the design equation. The fit solves for all of the polynomial coefficients and shifts of the data relative to the global $X Y$ origin. The physical central coordinate of the plano surface is calculated through an implementation of Welzl's algorithm to find the minimum bounding circle for the edge of the data. ${ }^{29}$ In this way, the shift in the GRIN profile is referenced to the physical center of the plano surface on the lens to within the spatial accuracy of acquired data. Finally, the design is subtracted from the fit GRIN profile so that lateral and axial shift of the GRIN profile can be determined.

\section{Results}

Raman measurement mapping of index of refraction is verified by Pulfrich refractometry for the homogeneous copolymer samples. Using the mapping of Raman spectrum to index of refraction, GRIN profile along the plano surface of the lens is presented, and GRIN profile deviations are calculated.

\subsection{Comparing Raman Measurement with Pulfrich Refractometry}

The Pulfrich refractometer has accuracy of $3.2 \times 10^{-5}$ index of refraction. ${ }^{1}$ Eleven homogeneous samples are measured, stepping in volume percentage of PS by $10 \%$. Two of the eleven samples, those containing pure PMMA and pure PS, are homopolymers, and the other nine are copolymerized samples.

Three data points at different locations on each homogeneous sample are taken with the Raman microscope to account for unwanted material variation within a reference measurement. The Raman-measured spectral weight coefficients of the two materials in each sample are used for the weighted average of the index of refraction of the two materials [Eq. (4)]. The weights are applied to the homogeneous PS and PMMA index of refraction measurements. This weighted average defines the index of refraction reported in the trend for the Raman data.

Pulfrich refractometry reports index of refraction directly for each sample, and error bars for that measurement are presented for comparison. The maximum departure of the Raman measurement from linear is $4 \times 10^{-3}$ index of refraction, and the maximum departure of the Pulfrich measurement from linear is $1 \times 10^{-3}$ index of refraction (Fig. 4). In this way, reference measurements directly map index of refraction to the Raman data.

(a)

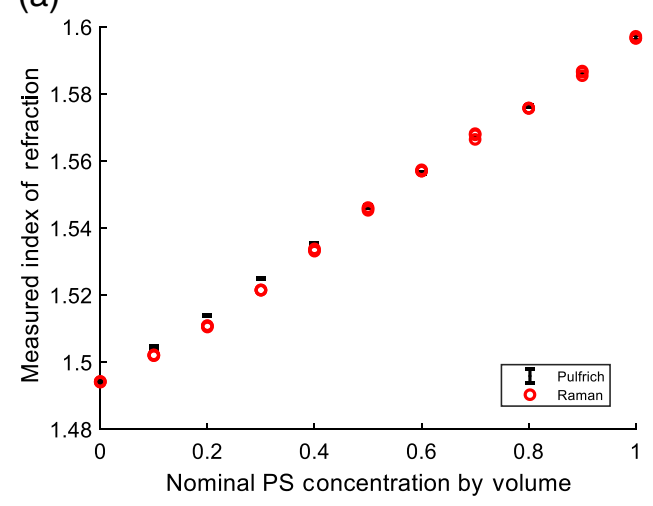

(b)

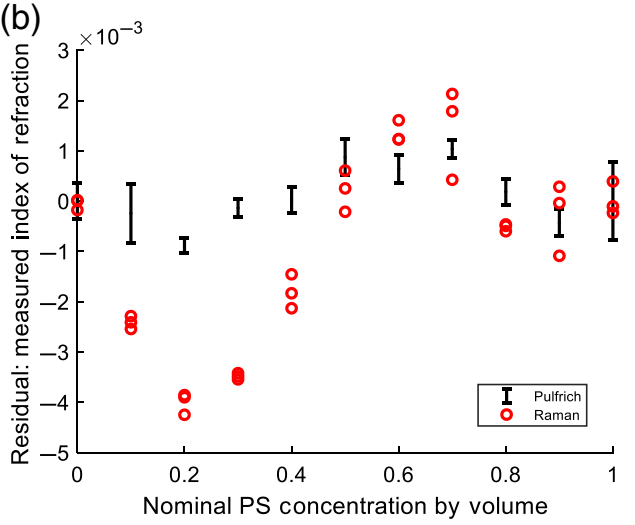

Fig. 4 Raman microscopy method to report index of refraction verified with Pulfrich refractometry. The trend is reported as a function of fabricated nominal volume fraction of PS for each of the 11 samples. (a) The data for each measurement method and (b) all data with the ideal linear trend subtracted from them are presented to more easily see a residual departure from linear. 


\subsection{Raman Microscopy of GRIN Lens}

The fabricated GRIN lens was designed to have a rotationally symmetric index of refraction profile (Fig. 3). Previous lenses were destructively measured; however, this sample was left intact to perform the Raman measurement. Raman confocal microscopy is used to scan the plane $z=0$ to measure any shift in the GRIN profile with respect to the plano surface aperture of the lens.

The design GRIN profile at the surface of the lens varies around 1.57 and 1.58 index of refraction. The reference measurements with the Pulfrich refractometer above show that the Raman spectral coefficient map to index of refraction is precise to within $1 \times 10^{-3}$ of index of refraction for these design values. The microscope is scanned in two dimensions over the plano surface of the PMMA/PS GRIN lens to gather the Raman spectra and map the index of refraction on that surface (Fig. 5). The scan covers a $24 \mathrm{~mm} \times 24 \mathrm{~mm}$ area, gathering $61 \times 61$ equally spaced data points and rejecting data at points outside the edge of the sample. This serves as built-in lateral sample registration to the scan. A few points within the sample region are rejected due to poor spectrum fit from low signal-to-noise ratio or cosmic ray contamination.

The data are fit to the designed GRIN profile equation [Eq. (5)] and compared to the design profile (Fig. 6). A shift in index of refraction along the $z$ axis (perpendicular to $x, y$; a difference in $n_{0}$ term) of $1.5 \times 10^{-3} \pm 3 \times 10^{-4}$ is observed between the GRIN data fit and design profile. Based on the design, the linear change in index of refraction along the $z$ axis is

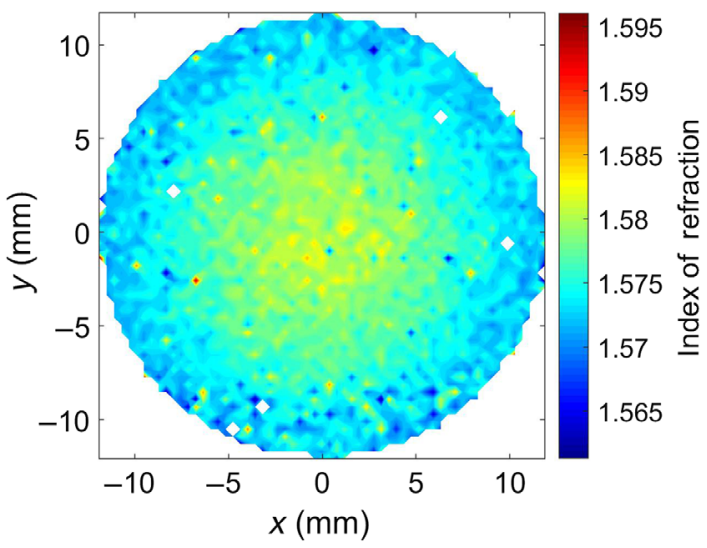

Fig. 5 Raw index of refraction data on the plano surface of the PMMA/PS GRIN lens. White data points indicate no data or spurious spectral measurements. The SA covers $24 \mathrm{~mm} \times 24 \mathrm{~mm}$ area, gathering $61 \times 61$ equally spaced data points. The microscope focal spot diameter for measurement is $1.7 \mu \mathrm{m}$.

(a)

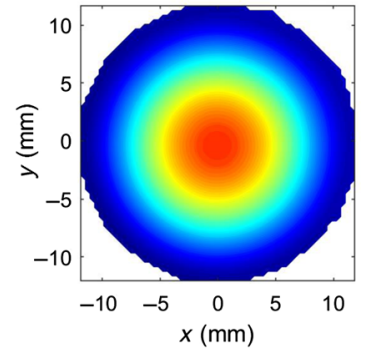

(b)

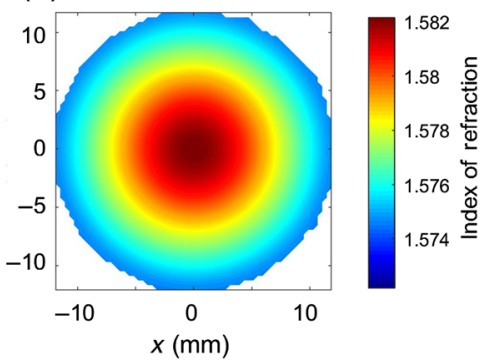

(c)

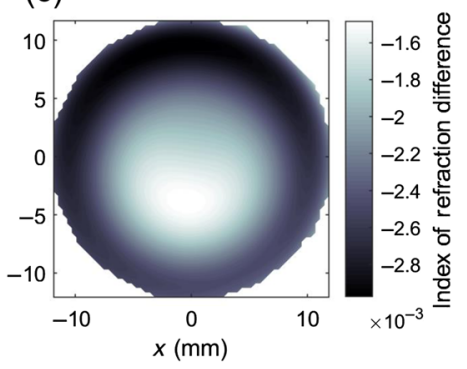

Fig. 6 (a) GRIN profile fit to the data and (b) the as-designed profile are plotted with the same scale, and (c) the difference figure of the design from the data is plotted with a different scale. All plots share the same $x$ and $y$ coordinates. 
$-2.2 \times 10^{-3} \mathrm{~mm}^{-1}$; the amount of index of refraction shift equates to a GRIN profile shift of $680 \mu \mathrm{m}$ in $z$ with respect to the desired position.

The MATLAB fitting toolbox effectively accounts for the low-order polynomial fit coefficient, but cannot fit higher order polynomial coefficients. Level of noise limits certainty, and higher order profile terms are not reliable. The decenter terms $\delta x$ and $\delta y$ from the fit show GRIN profile shift in $x$ and $y$ is $<400 \mu \mathrm{m}$, which is also the precision of centering the data to the lens. The error in the GRIN profile shows a slight shift of higher order radial contributions of the design, not normal tilt and decenter of the GRIN within the lens.

\section{Discussion}

The result is the first direct measurement of index of refraction profile on the surface of this GRIN lens. Previous measurements were only able to measure the total change in index of refraction by the optical path difference through the sample. Raman microscopy is correlated with the Pulfrich refractometry using homogeneous sample measurements. By taking reference measurements of homogeneous mixed PS/PMMA copolymer, the index of refraction can effectively be mapped. The reference measurement is made to within $1 \times 10^{-3}$ index of refraction for values relevant to the GRIN lens measurement. With a finer step in reference sample polymer composition, a finer precision reference mapping will be possible.

Sampling the plano surface more finely may result in finer spatial definition of the edge, and therefore improved spatial referencing for tilt and decenter measurements of the GRIN. With further calibration of the microscope focal volume, the method may be extended to measure GRIN at a curved optical surface. This could be attempted in following experiments.

Unlike interferometry, Raman spectroscopy may measure large absolute index of refraction departure from design without destroying the measurement feasibility. This makes Raman spectroscopy a faster nondestructive test method for GRIN optic fabrication.

The PMMA/PS copolymer GRIN lens is shown to be a suitable candidate for index of refraction mapping through Raman microscopy. The Raman measurement is correlated with Pulfrich refractometry, and the index of refraction map of the GRIN lens is laterally aligned to the design specification to within the lateral resolution of the measurement. The GRIN profile is found to be axially displaced by $680 \mu \mathrm{m}$ in $z$. Higher spatial resolution for measurement may be necessary to determine the GRIN profile shift if tighter tolerance specifications are required.

\section{Acknowledgments}

The authors thank Joe Malone for his help in taking the Raman microscope measurements. The authors declare no conflicts of interest in this work or in the publication of this work. All authors consent to the publication of this work.

\section{References}

1. K. Fang, "Design, fabrication and characterization of polymer gradient-index (GRIN) material," PhD Thesis, University of Rochester (2016).

2. T. Uchida et al., "Optical characteristics of a light-focusing fiber guide and its applications," IEEE J. Quantum Electron. 6, 606-612 (1970).

3. M. van Buren and N. A. Riza, "Foundations for low-loss fiber gradient-index lens pair coupling with the self-imaging mechanism," Appl. Opt. 42, 550-565 (2003).

4. H. Melkonyan et al., "Gradient-index optical fiber lens for efficient fiber-to-chip coupling," Opt. Exp. 25, 13035-13045 (2017).

5. S. Guo et al., "Gradient-index lens rod based probe for office-based optical coherence tomography of the human larynx," J. Biomed. Opt. 14(1), 014017 (2009).

6. S. Y. Leigh and J. T. C. Liu, "Multi-color miniature dual-axis confocal microscope for pointof-care pathology," Opt. Lett. 37, 2430-2432 (2012).

7. S. Ohmi et al., "Gradient-index rod lens made by a double ion-exchange process," Appl. Opt. 27, 496-499 (1988). 
8. A. Visconti, "Design and fabrication of large diameter gradient-index lenses for dual-band visible to short-wave infrared imaging applications," $\mathrm{PhD}$ Thesis, University of Rochester (2015).

9. C. Wang and D. L. Shealy, "Design of gradient-index lens systems for laser beam reshaping," Appl. Opt. 32, 4763-4769 (1993).

10. Y. Koike, H. Hidaka, and Y. Ohtsuka, "Plastic axial gradient-index lens," Appl. Opt. 24, 4321-4325 (1985).

11. K. Fuerschbach, "Freeform, $\phi$-polynomial optical surfaces: optical design, fabrication and assembly," PhD Thesis, University of Rochester (2014).

12. S. D. Campbell et al., "Three-dimensional gradient-index optics via inkjet-aided additive manufacturing techniques," in IEEE Int. Symp. Antennas and Propagation USNC/URSI National Radio Science Meet., pp. 605-606 (2015).

13. Y. F. Chao and K. Y. Lee, "Index profile of radial gradient index lens measured by imaging ellipsometric technique," Jpn. J. Appl. Phys. 44, 1111-1114 (2005).

14. C. K. Carniglia, "Ellipsometric calculations for nonabsorbing thin films with linear refractive-index gradients," J. Opt. Soc. Am. A 7, 848-856 (1990).

15. D. Lin et al., "One-dimensional gradient-index metrology based on ray slope measurements using a bootstrap algorithm," Opt. Eng. 52(11), 112108 (2013).

16. D. Lin and J. R. Leger, "An iterative approach to measuring two-dimensional gradient-index profiles based on external measurements of laser beam deflection," Proc. SPIE 9576, 95760G (2015).

17. M. A. Sekh et al., "Measurement of gradient index profile using deflectometry," Opt. Commun. 306, 145-149 (2013).

18. F. J. Purcell and J. M. Bello, "Fluorescence-free Raman spectra of polymers," Proc. SPIE 1336, 135-143 (1990).

19. C. Hu et al., "Observation of mutual diffusion of macromolecules in PS/PMMA binary films by confocal Raman microscopy," Soft Matter 8, 4780-4787 (2012).

20. J. P. Tomba, "Comment on 'Observation of mutual diffusion of macromolecules in PS/ PMMA binary films by confocal Raman microscopy' by C. Hu, X. Chen, J. Chen, W. Zhang and M. Q. Zhang, Soft Matter, 2012, 8, 4780," Soft Matter 12, 4510-4513 (2016).

21. J. P. Tomba, L. M. Arzondo, and J. M. Pastor, "Depth profiling by confocal Raman microspectroscopy: semi-empirical modeling of the Raman response," Appl. Spectrosc. 61(2), 177-185 (2007).

22. N. J. Everall, "Modeling and measuring the effect of refraction on the depth resolution of confocal Raman microscopy," Appl. Spectrosc. 54(6), 773-782 (2000).

23. A. Žukauskas et al., "Tuning the refractive index in 3D direct laser writing lithography: towards GRIN microoptics," Laser Photonics Rev. 9(6), 706-712 (2015).

24. M. Brindza et al., "Refractive index of nanolayered polymeric optical materials," in Laser Appl. Photonic Appl., JWA73, Optical Society of America (2011).

25. L. Sisken et al., "Evidence of spatially selective refractive index modification in $15 \mathrm{gese}_{2}-45 \mathrm{as}_{2} \mathrm{Se}_{3}-40$ pbse glass ceramic through correlation of structure and optical property measurements for grin applications," Opt. Mater. Express 7, 3077-3092 (2017).

26. G. P. Lindberg et al., "Raman and CT scan mapping of chalcogenide glass diffusion generated gradient index profiles," Proc. SPIE 9822, 98220W (2016).

27. G. P. Lindberg et al., "Methods of both destructive and non-destructive metrology of GRIN optical elements," Proc. SPIE 9451, 94511 S (2015).

28. B. D. Beier, R. G. Quivey, Jr., and A. J. Berger, "Identification of different bacterial species in biofilms using confocal Raman microscopy," J. Biomed. Opt. 15(6), 066001 (2010).

29. A. Semechko, "Exact minimum bounding spheres and circles," GitHub repository, 2020, https://github.com/AntonSemechko/Bounding-Spheres-And-Circles.

Nicholas S. Kochan received his BS degree in optics and his MS degree in technical entrepreneurship and management from the University of Rochester in 2017 and 2019, respectively. He is a PhD student at the University of Rochester Institute of Optics, studying optical system design and optical metrology. He is a member of SPIE. 
Greg R. Schmidt received his PhD in optical engineering in 2009 from the University of Rochester. He is a research professor at The Institute of Optics. His primary field of research is gradient index optics materials, modeling, and metrology. He also studies nonimaging optics for concentrating solar and illumination. He is a member of SPIE.

Andrew J. Berger received his BS degree from Yale in 1991 and his $\mathrm{PhD}$ from the Massachusetts Institute of Technology in 1998, both in physics. He has been a professor of optics and biomedical engineering at the University of Rochester since 2000. He is the author of more than 40 journal articles and has written 2 book chapters. His current research interests include in vivo vibrational spectroscopy of bone, estimation of organelle size distribution in single biological cells using angularly resolved elastic scattering, and spectroscopic enhancement of bruise contrast for better documentation of interpersonal violence.

Duncan T. Moore received his bachelor's degree in physics from the University of Maine, his master's degree in optics from the University of Rochester, and his PhD in optics in 1974. Currently, he is the vice provost of entrepreneurship and the Kingslake professor of optical engineering, professor of business administration at the University of Rochester. He has extensive experience in the academic, research, business, and governmental arenas of science and technology. He is an expert in gradient-index optics, concentrating solar optics, and the manufacture of optical systems. He is a fellow of SPIE and received the SPIE Gold Medal in 2006. 